\section{P278 STRATEGIES DEVELOPED BY MINISTRY OF HEALTH OF BRAZIL TO INCREASE HIV DIAGNOSIS SINCE 2012}

Pâmela Gaspar*, Mariana Villares, Alisson Bigolin, Regina Comparini, José Neto, Adele Benzaken, Gerson Fernando Pereira. Ministry of Health of Brazil, Department of Surveillance, Prevention and Control of Sexually Transmitted Infections, HIVIAIDS and Viral Hepatitis, Brasilia, Brazil

\subsection{6/sextrans-2019-sti.403}

Background In 2012, 69\% of people living with HIV (PLHIV) in Brazil knew their HIV status. In contrast 731,000 thousand people were diagnosed until 2017, corresponding $84 \%$ of the current 866,000 PLHIV in the country. This study aims to describe the actions developed to increase HIV diagnosis in Brazil since 2012.

Methods HIV rapid test (RT) in primary care settings has been being the key strategy to enhance HIV diagnosis in Brazil. Therefore, it was crucial to increase the number of healthcare professionals capable to perform RT. Considering the need of an alternative training method to on-site, the Ministry of Health $(\mathrm{MoH})$ has been offering the free distance learning course called TELELAB. In addition, the National Program of External Quality Assessment for RT (EQA-RT) was introduced to monitor the quality of the RT performed by those healthcare professionals. The use of oral fluid RT was also implemented to develop outreach strategies to promote HIV testing.

Results There was a $270 \%$ increase in the number of RT acquired and distributed by the MoH between 2012 and 2018, totalizing 14 million tests in 2018. In 2018, 15,146 professionals were certified online by TELELAB on the HIV diagnosis course, leading to a total of 42,026 professionals since 2012. Eighteen rounds of EQA-RT were conducted, with around 90\% approval at the last one. Finally, since 2014, outreach strategies performed 174,000 oral fluid RT by peer-topeer testing by non-governmental organizations focusing on key-population.

Conclusion The synergy of the strategies described was crucial to ensure the reliability and credibility of the RT results and to reach the population not reached previously by the standard testing, resulting in 15\% increase of HIV diagnosis in the period of 2012-2017. Since 2018, the $\mathrm{MoH}$ started the free of charge distribution of HIV self-testing as a new tool to continuously improve diagnosis strategies.

Disclosure No significant relationships.

\section{P279 DEVELOPMENT AND VALIDATION OF SEXUALLY TRANSMITTED INFECTIONS DECISION MODELLING SOFTWARE IN COOPERATION WITH POLICY MAKERS}

${ }^{1}$ Fabian Sailer, ${ }^{2}$ John Saunders* ${ }^{3}{ }^{3}$ Greta Rait, ${ }^{1}$ Rachael Hunter. ${ }^{1}$ Research Department of Primary Care and Population Health, London, UK; ${ }^{2}$ University College London, NIHR Health Protection Research Unit in Blood Borne and Sexually Transmitted Infections, London, UK; ${ }^{3}$ University College London, Research Department of Primary Care and Population Health, London, UK

\subsection{6/sextrans-2019-sti.404}

Background HIV and other sexually transmitted infections (STIs) do not operate in isolation, particularly as people with risk-taking sexual behaviour may be co-infected. In this complex landscape, policy makers are limited by resource constraints while trying to find optimal coverage solutions. Disease modelling could help in this context. We aim to develop a user-friendly modelling software examining several STIs and HIV simultaneously, as we are unaware of any multi-STI decision support tools currently available.

Methods We developed STI modelling software using the programming language Java, consisting of several models and a graphical user interface (UI). The models were drafted based on literature reviews and subsequently refined by experts, e.g. STI clinicians and policy makers. All models were internally and externally validated. The UI was developed with UI development experts and policy makers.

Results Separate disease models, which describe the progression of chlamydia, gonorrhoea, HIV, syphilis, and their sequelae are included in the software. Sexual network models are used to describe the formation and dissolution of partnerships and thereby the occurrence of sexual contacts. Four different network models are included in the software. The clinical pathway models describe interventions, for example screening or STI treatment and reflect the current UK setting. All the models are interacting, individual-based discrete event simulations and have been validated using sensitivity analyses and publicly available data sources. The UI has been validated by policy makers.

Conclusion With this modelling software policy makers can compare both existing and hypothetical intervention options in regards to their costs and consequences. All the parameters, formulas, model structures, and clinical pathways are editable. The software is flexible and usable in different settings and contexts. It can be and updated if needed, e.g. if medical knowledge changes. By adapting parameters which describe treatment pathways the software could be used in non-UK settings.

Disclosure No significant relationships.

\section{P280 USE OF MOBILE HEALTHCARE UNITS IN RAPID TEST EVENTS IN COMBATING SEXUALLY TRANSMITTED INFECTIONS}

${ }^{1}$ Ana Amélia Bones*, ${ }^{2}$ Mcarthur Barrow, ${ }^{3}$ Carlos Andres Casas, ${ }^{4}$ Marina Dias ${ }^{4}$ Gabriela Storck, ${ }^{1}$ Silvio Cazella, ${ }^{5}$ Airton Stein. ${ }^{1}$ Health Federal University from Porto Alegre, Health Informatics Post Graduation Program, Porto Alegre, Brazil; ${ }^{2}$ Municipal Secretary of Health of Porto Alegre, Primary Health Care Trainee Program, St. Philip, Barbados; ${ }^{3}$ Health Federal University from Porto Alegre, Medicine Academic, Bogota, Colombia; ${ }^{4}$ Municipal Secretary of Health of Porto Alegre, Departament of Sexually Transmitted Infections, Porto Alegre, Brazil, ${ }^{5}$ Health Federal University from Porto Alegre, Health Science Post Graduation Program, Porto Alegre, Brazil

\subsection{6/sextrans-2019-sti.405}

Background Social engagement used as a tool for providing access to information and testing for Sexually Transmitted Infections (STIs) during events can be a promising strategy for reaching different population groups in public settings. The use of Rapid Tests (RT) for syphilis offers an unique opportunity to facilitate care in response to the syphilis epidemic. The objective of this study is to evaluate the epidemiological profile of people with positive RT for syphilis observed during RT events in Porto Alegre City, Brazil.

Methods Cross-sectional study using a time series approach in all RTs carried out in Mobile unit RT events in 2018, through data mining, with WEKA software.

Results The events were held in public spaces, such as squares and parks, included roundtable discussions, presentations, condom distribution, RTs for STIs, individual medical care with possibility of prescription and application of medication. 
Among 4156 RTs conducted, 626(15\%) were positive for syphilis. The characteristics of the population tested were: 2003(48\%) women, 374(9\%) homo/bisexual, 710(17\%) under 25 years and 1001(24\%) elderly. In the subgroup with positive syphilis RTs, 250(39.9\%) were women, 29(4.9\%) homo/ bisexual, 44(7\%) under 25 years, 43(6.8\%) positive RT for HIV and 43(6.8\%) for Hepatitis C. Although the RTs for syphilis did not confirm diagnosis, the framework in place to deal with positive tests provides same-day medical consultation to evaluate the need for immediate treatment at the event site and referral for follow-up or continued treatment at the Primary Healthcare level.

Conclusion Social interaction with the public during RT using a mobile healthcare units in places intended for leisure sought to increase awareness and access to people with undiagnosed STIs. Ensuring that specific subgroups of the general population have access to testing and medical consultation were shown to be important points when applying point of care tests. These aspects of community testing should be evaluated in future research.

Disclosure No significant relationships.

\section{P281 REACHING OUT TO MSM WHO FLED FOR WAR AND LGBT OPPRESSION FOR HIV AND STI TESTING, HBV VACCINATION AND COUNSELLING}

${ }^{1}$ Wim Zuilhof, ${ }^{2}$ Hanna Bos*. ${ }^{1}$ SOA AIDS Nederland, MSM Programme, Amsterdam, Netherlands; ${ }^{2}$ SOA AIDS Nederland, Amsterdam, Netherlands

\subsection{6/sextrans-2019-sti.406}

Background The Netherlands has had a large influx of MSM refugees in recent years. Soon after arriving many of them start dating other MSM for sexual hook-ups, while insufficiently aware of risks that sex between men entails for HIV/ STI. They are also unfamiliar with existing facilities for HIV/ STI testing, HBV-vaccination and HIV-care in the Netherlands. Methods In 2017 and 2018, MSM refugees were invited for meet-ups in Amsterdam and three other cities, where information and counseling about STI and HIV and how to prevent these was given in multiple languages. HIV and STI tests and vaccination for $\mathrm{HBV}$ were also offered on the spot. Only refugees were allowed in, thus creating a safer space atmosphere. Those tested and/or vaccinated received follow-up appointments on regular office hours for test results, further HIV/STI care or completion of HBV vaccinations. These meet-ups were possible through very close cooperation between self-organizations of migrant MSM, regional Public Health Services, gay bars and a national NGO.

Results In 2017 and 2018 a total of 858 MSM refugees attended 8 meet-ups on which $223 \mathrm{HIV} / \mathrm{STI}$ tests were done and $226 \mathrm{HBV}$-vaccinations were given. 43 STI's and $3 \mathrm{HIV}$ infections were found. A survey among visitors showed huge appreciation of the meet-ups. A large proportion of the visitors visited more than one meet up, thus showing appreciation for and trust in the organisers. One on one counselling of visitors by volunteers and health professionals during the meet ups showed lack of knowledge about HIV and STI and lack of behavioural skills among visitors and the need for development of behavioural interventions specifically for this group. Conclusion MSM refugees can be reached through gay community meet-ups for HIV/STI testing and HBV vaccination. Extra data have to be collected for the development of much needed behavioural interventions.

Disclosure No significant relationships.

\section{P283 THE ALBERTA 2016-2020 STBBI OPERATIONAL STRATEGY AND ACTION PLAN: COLLECTIVE IMPACT TO ADDRESS THE PROVINCIAL STI OUTBREAK}

${ }^{1}$ Cari Egan*, ${ }^{1}$ Christopher Wood, 'David Strong, ${ }^{2}$ Jennifer Gratrix, ${ }^{2}$ Petra Smyczek, ${ }^{3}$ Abhaya Prasad, ${ }^{3}$ Lindsay Rathjen. ${ }^{1}$ Alberta Health Services, Communicable Disease Control, Calgary, Canada; ${ }^{2}$ Alberta Health Services, STI Services, Edmonton, Canada; ${ }^{3}$ Alberta Health Services, Edmonton, Canada

\subsection{6/sextrans-2019-sti.407}

Background In April of 2016, a provincial sexually transmitted infections (STI) outbreak was declared in Alberta. In response to sharply rising rates, Alberta formed the Alberta Sexually Transmitted and Blood Borne Infection (STBBI) Network (ASN) and developed the 2016-2020 STBBI Operational Strategy and Action Plan (OSAP). The aim of the STBBI OSAP is to transform STBBI care in Alberta by leveraging engagement and commitment from within the ASN to achieve collective impact and develop wrap around shared care models through formalized interagency collaboration.

Methods ASN has grown to include over 500 stakeholders representing approximately 200 community-based, provincial and federal organizations including communities most affected by STBBI (Indigenous, LGBTQ2+, immigrants, and street involved clients). The OSAP comprises 74 recommendations that focus on five pillars of client-centred STBBI care: (1) prevention, (2) testing/screening, (3) treatment, (4) linkage to care, and (5) stigma. Seven working groups designed 15 project concepts with endorsement from the Steering Committee to optimize achievement of prioritized recommendations.

Results Implementation of STBBI OSAP priority recommendations is still underway in 2019. Some prioritized action items include: PrEP guidelines and training program for designated prescribers; a validation study of self-collected gonorrhea/chlamydia NAAT specimens to improve access to low barrier testing opportunities; expedited partner therapy; expanded Hepatitis C treatment access in Indigenous communities; education for primary care providers to increase access and linkage to STBBI care; and formalizing interagency collaboration through the development of STBBI wrap around shared care model demonstration projects.

Conclusion The STBBI OSAP is an innovative, comprehensive, and collaborative intersectoral approach to address the high burden of STBBI in Alberta and to strengthen the provincial outbreak response. The strength of this provincial initiative is the engagement and collaboration amongst ASN stakeholders to achieve long lasting impact and sustainability of both systemic and operational interventions.

Disclosure No significant relationships. 